\title{
ON SOME NONCOERCIVE VARIATIONAL INEQUALITIES CONTAINING DEGENERATE ELLIPTIC OPERATORS
}

\author{
VY KHOI LE'
}

(Received 10 September, 1999; revised 23 May, 2001)

\begin{abstract}
We are concerned with the solvability of variational inequalities that contain degenerate elliptic operators. By using a recession approach, we find conditions on the boundary conditions such that the inequality has at least one solution. Existence results of LandesmanLazer type for a nonsmooth inequality and a resonance problem for a weighted $p$-Laplacian are discussed in detail.
\end{abstract}

\section{Introduction-Background}

The present paper is concerned with some solvability results for equations and variational inequalities that contain degenerate elliptic operators. Our interest is in problems at resonance or problems with boundary conditions other than the Dirichlet condition, which can be formulated as variational inequalities of the form

$$
\left\{\begin{array}{l}
\int_{\Omega} A(x, \nabla u)(\nabla v-\nabla u) d x+\int_{\Omega} g(x, u)(v-u) d \mu+j(v)-j(u) \\
\quad \geq\langle f, v-u\rangle, \forall v \in K \\
u \in K
\end{array}\right.
$$

Here $A$ is a degenerate nonlinear elliptic operator that corresponds to a weight function $m(x)$ where $d \mu=m(x) d x$. We have also that $A: \Omega \times \mathbb{R}^{N} \rightarrow \mathbb{R}^{N}$ satisfies the coercivity (ellipticity) condition $A(x, v) v \geq \alpha m(x)|v|^{p}$, and the growth condition

$$
|A(x, v)| \leq \beta m(x)|v|^{p-1}, \quad \text { a.e. } x \in \Omega, \forall v \in \mathbb{R}^{N},
$$

where $g(x, u)$ is a lower order perturbing term, $f$ belongs to the dual space, $K$ is a closed convex set, and $j$ is a convex, lower semicontinuous functional. Those

\footnotetext{
'Department of Mathematics and Statistics, University of Missouri-Rolla, Rolla, MO 65409, USA; e-mail: vy@umr.edu.

(C) Australian Mathematical Society 2003, Serial-fee code 1446-1811/03
} 
variational inequalities are, in general, noncoercive. Our goal here is to examine existence results for nonlinear, noncoercive, degenerate, elliptic equations and variational inequalities of the form (1.1).

The variational inequality (1.1) is the weak form of various differential equations with obstacles or unilateral conditions. For example, the obstacle problem

$$
\left\{\begin{array}{c}
-\operatorname{div}(A(x, \nabla u))+g(x, u) \geq 0, \\
u \geq \psi, \\
{[-\operatorname{div}(A(x, \nabla u))+g(x, u)](u-\psi)=0 \text { in } \Omega,}
\end{array}\right.
$$

with $u=0$ on $\partial \Omega$ ( $\psi$ is a given function on $\Omega$ ), has (1.1) as a weak formulation with $j=0$ and $K=\{v \in X: v \geq \psi$ in $\Omega\}$ (see, for example, [9,25] for the derivation). On the other hand, the equation

$$
-\operatorname{div}(A(x, \nabla u))+g(x, u)=0 \text { in } \Omega,
$$

with the unilateral boundary condition

$$
u \geq \psi, \quad \frac{\partial u}{\partial n} \geq 0, \quad(u-\psi) \frac{\partial u}{\partial n}=0 \quad \text { on } \partial \Omega,
$$

( $\psi$ is a given function on $\partial \Omega$ ) can be written in the weak form (see [18]) as the inequality (1.1) with $j=0$ and $K=\{v \in X: v \geq \psi$ on $\partial \Omega\}$, or $K=X$ and

$$
j(v)= \begin{cases}0 & \text { if } v \geq \psi \text { on } \partial \Omega \\ \infty & \text { otherwise }\end{cases}
$$

In the above examples, $X$ is an appropriately weighted Sobolev space, chosen based on the above ellipticity and the growth conditions of the principal operator $A$. The degeneracy of $A$ comes from the dependence of $A$ on $\nabla u$ and also from the weight function $m(x)$ in the above ellipticity condition. In fact, the value of $A(x, \nabla u)$ may vanish at the points where $\nabla u=0$ or $m(x)=0$.

Degenerate equations and inequalities, in particular those containing the $p$-Laplacian, have many applications in nonlinear elasticity, fluid mechanics (flow through porous media), glaciology, petroleum extraction, etc. (see for example [13,15,22, 33 ] and the references therein). Recent applications of degenerate equations and inequalities to sandpile evolutions, river networks and problems in superconductivity have been investigated in $[7,38-40]$.

The study of degenerate elliptic equations seems to have been initiated by the pioneering work of Murthy and Stampacchia [35], where equations of the form

$$
\int_{\Omega}\left[\left(a_{i j} u_{x_{i}}+d_{j} u\right) \phi_{x_{j}}+\left(b_{i} u_{x_{i}}+c u\right) \phi\right] d x=\int_{\Omega} F \phi, \quad \forall \phi \in C_{0}^{\infty}(\Omega)
$$


were studied. Here the $a_{i j}$ 's satisfy a degenerate elliptic condition

$$
\sum_{i, j} a_{i j}(x) \xi_{i} \xi_{j} \geq m(x)|\xi|^{2},
$$

where $m(x) \geq 0$ may be 0 at points of $\Omega$. The paper was followed up by the works of Trudinger, Alvino, Trombetti, Ivanov, Mkrtycjan and others (see [2, 3, 23,43] and the references therein). Attention to degenerate equations has been recently renewed with the works of Drabek, Kufner, Leonardi, Nicolosi and others (see $[12,14,16$, $17,32,36]$ and the references therein). In those papers, several existence results for degenerate elliptic equations with Dirichlet boundary conditions were established by topological arguments and/or existence results for equations containing Leray-Lions operators. In [31], Le and Schmitt examined existence questions concerning positive solutions of degenerate nonlinear elliptic equations and variational inequalities by using topological tools such as the fixed point index and fixed point theorems.

However, in most of the cited papers, Dirichlet boundary conditions were considered, which implies the coercivity of the operator defined by the principal part, which consists of the highest order derivatives. In problems with resonance or with Neumann or unilateral boundary conditions, the principal operators are usually noncoercive. Those problems have not been investigated in detail so far. Because the kernel of the principal operator is nontrivial, a priori estimates are not immediately available, and topological methods, which have often been used before (see for example [31] and the references therein) are not directly applicable. We will consider in the present paper solvability conditions for these noncoercive problems with degenerate operators. We use for this purpose a recession approach, that is, we try to relate the solvability of noncoercive equations or inequalities with behaviour at infinity of certain corresponding operators and functionals. The recession method seems to have first been used by Hess in [21] to prove existence results for noncoercive equations.

We do not assume here that the problems have variational structures; hence they may not come from minimisation problems. Since our existence results for degenerate equations and variational inequalities can be derived as consequences of some general abstract results, we first present an existence theorem for general variational inequalities in Banach spaces. Afterwards, the general arguments and results are used to investigate the solvability of variational inequalities that contain nonlinear degenerate operators, such as the weighted $p$-Laplacian.

The paper is organised as follows. We begin with some abstract results in Section 2 and after that, in Section 3, apply them to examples of degenerate equations and inequalities. In Section 2, we introduce a basic property, called Property (P), of a class of noncoercive problems. The Property $(\mathrm{P})$ for variational inequalities that we define here is motivated by certain related concepts in $[29,30]$ and [5]. We next show in Theorem 1 that, under some compatibility conditions, problems with Property (P) 
are solvable.

Section 3 is devoted to existence results for variational inequalities containing degenerate elliptic operators. The problems are formulated in appropriately weighted Sobolev spaces. The perturbing terms could have general, subcritical rates of growth at infinity, not necessarily sublinear, as usually assumed in problems with resonance (by using recession methods). Also, the perturbing terms could depend on the gradient of the unknown function and the problems may not have variational structures. The variational methods usually used are therefore not applicable. We establish in this section existence results of Landesman-Lazer type for nonsmooth problems that contain general degenerate elliptic operators (Section 3.1). Section 3.2 is concerned with the solvability of a resonance problem for a variational inequality containing a weighted $p$-Laplacian.

The abstract existence results here are adaptations and generalisations of those presented in $[29,30]$ and hence will only be outlined in Section 2. On the other hand, the justifications of the abstract and general conditions in Theorem 1 and Proposition 1 for our particular degenerate equations and inequalities in Section 3 require nontrivial arguments and calculations and new properties and estimates in weighted Sobolev spaces. The recession methods and results in Section 3 seem to be new for problems concerning degenerate equations and inequalities. They also improve and generalise a number of results in this field.

\section{An abstract existence result}

Let $X$ be a reflexive Banach space with norm $\|\cdot\|$, dual $X^{*}$ and dual pairing $\langle\cdot, \cdot\rangle$. Assume that $K$ is a closed, convex subset of $X$ and $j: X \rightarrow \mathbb{R} \cup\{\infty\}$ is a convex, lower semicontinuous functional. In what follows, $\rightarrow$ is used to denote weak convergence in $X$ or weak* convergence in $X^{*}$. For simplicity, we suppose throughout the paper that $0 \in K$ and $j(0)=0$ (in particular, $D(j) \cap K \neq \emptyset$ ). We assume that $A: X \rightarrow X^{*}$ is a bounded, continuous and pseudo-monotone operator (that is, if $x_{n} \rightarrow x$ in $X$ and $\lim \sup \left\langle A\left(x_{n}\right), x_{n}-x\right\rangle \leq 0$, then $\lim \inf \left\langle A\left(x_{n}\right), x_{n}-v\right\rangle \geq\langle A(x), x-v\rangle, \forall v \in X$ (see [33] or [10])). Also, $A$ is bounded by polynomial functions of $\|u\|$, that is, there exist positive numbers $\beta, c$ and $d$ such that

$$
\|A(u)\| \leq c\|u\|^{\beta}+d,
$$

for all $u \in X$. We are concerned in this section with existence results for variational inequalities of the form

$$
\left\{\begin{array}{l}
\langle A(u), v-u\rangle+j(v)-j(u) \geq\langle f, v-u\rangle, \quad \forall v \in K, \\
u \in K,
\end{array}\right.
$$


where $f \in X^{*}$. Motivated by the definitions in [29] and [5], we consider the following property of a class of noncoercive problems.

DefinItion 1. The pair $(A, j)$ is said to have Property (P) on the set $K$ if the following condition holds:

If $\left\{u_{n}\right\}$ is a sequence in $K$ such that

$$
\begin{gathered}
\left\|u_{n}\right\| \rightarrow \infty, \\
\limsup \frac{\left\langle A u_{n}, u_{n}\right\rangle}{\left\|u_{n}\right\|^{\lambda}} \leq 0, \quad \forall \lambda>1
\end{gathered}
$$

and

$$
\frac{u_{n}}{\left\|u_{n}\right\|} \rightarrow w
$$

with $w$ satisfying

$$
\left\|u_{n}\right\| \leq\left\|u_{n}+\lambda w\right\|, \quad \forall n, \forall \lambda \in \mathbb{R},
$$

then there exists $u_{0} \in K \cap D(j)$ such that

$$
\limsup \left[\frac{\left(A u_{n}, u_{n}-u_{0}\right)+j\left(u_{n}\right)}{\left\|u_{n}\right\|}\right]>0 .
$$

Note that condition (2.6) is, in some sense, the orthogonality between $w$ and $u_{n}$ in the Banach space $X$. We have the following existence result for (2.2).

THEOREM 1. Assume that $(A, j)$ has Property $(\mathrm{P})$ on $K$ and the following compatibility condition holds:

If $w \in \mathrm{rc} K$ is such that there exists a sequence $\left\{u_{n}\right\} \subset K$ satisfying (2.3)-(2.5), and moreover,

$$
\lim \sup \frac{\left\langle A\left(u_{n}\right), u_{n}\right\rangle}{\left\|u_{n}\right\|}+j_{\infty}(w) \leq\langle f, w\rangle
$$

then

$$
\begin{gathered}
-w \in \operatorname{rc} K, \\
j_{\infty}(w) \leq\langle f, w\rangle \leq \min \left\{-j_{\infty}(-w), 0\right\},
\end{gathered}
$$

and there exists a subsequence $\left\{u_{n_{k}}\right\} \subset\left\{u_{n}\right\}$ such that

$$
\left\langle A\left(u_{n_{k}}\right), w\right\rangle=0, \quad \forall k,
$$

then the variational inequality (2.2) has a solution.

Here rc $K$ denotes the recession cone of $K, \operatorname{rc} K=\bigcap_{t>0} t K$ and $j_{\infty}$ is the recession functional associated with $j$, defined by $j_{\infty}(v)=\lim _{t \rightarrow \infty} j(t v) / t(v \in X)$. We refer to $[42]$ (the finite-dimensional case) or $[8,28,41]$ (the infinite-dimensional case with 
possible nonconvex sets or functionals) for basic properties of rc $K$ and $j_{\infty}$. In particular, rc $K$ is the closure of the set $\left\{w \in X: \exists t_{x}>0, a_{x} \in K: a+t w \in K, \forall t \geq\right.$ $\left.t_{x}\right\}$. We also note that if $f=0$ and $j=0$, then in (2.4) we can replace $p>1$ by the weaker condition $p>0$.

Although not stated explicitly before in this form, similar results to Theorem 1 for minimisation problems were established in [29]. Our proof of Theorem 1 follows the same lines as in [29, Theorems 2.5 and 4.4] and is therefore omitted.

To conclude this section, we present a sufficient condition on $A$ such that $(A, j)$ has Property $(\mathrm{P})$. Although more restrictive, the condition is easier to verify and is satisfied by several usual nonlinear operators. Proposition 1 will be applied later to show that certain degenerate elliptic operators have Property (P). Its proof is straightforward and is thus omitted.

Proposition 1. Assume $A=L+G$, where $L$ is homogeneous of degree $p-1$ for some $p>1$, that is,

$$
L(\lambda u)=\lambda^{p-1} L(u), \quad \forall \lambda \geq 0, \forall u \in X,
$$

and $G$ is completely continuous and satisfies

$$
\liminf _{\|u\| \rightarrow \infty} \frac{\langle G(u), u\rangle}{\|u\|^{p}} \geq 0 .
$$

Moreover, the functional $\Phi$ defined by $\Phi(u)=\langle L(u), u\rangle, \forall u \in X$, is nonnegative, weakly lower semicontinuous and

$$
\Phi(u-w)=\Phi(u), \quad \forall u \in X, \forall w \in \Phi,
$$

$(\Phi=\{w \in X: \Phi(w)=0\})$ and there exists a completely continuous mapping $P: X \rightarrow \mathbb{R}^{+}$such that

$$
\Phi(u)+P(u) \geq\|u\|^{p}, \quad \forall u \in X .
$$

Under these conditions, $(A, j)$ has Property (P) for any $j$ convex and lower semicontinuous.

REMARK 1. The approach used here is motivated by [29] and [30]. In these papers, we examined sufficient conditions such that noncoercive minimisation problems have minimisers. Although based on similar ideas, [29] and [30] aim at minimisation problems and equations with variational structure, while we concentrate here on those without variational structure, which is the case for elliptic equations and inequalities with degenerate operators. 


\section{Existence of solutions for unilateral degenerate elliptic problems}

\subsection{Existence results of Landesman-Lazer type for inequalities with degenerate} operators In this section, we apply the abstract results in Section 2 to existence issues concerning variational inequalities and equations that contain degenerate, nonlinear, elliptic operators. We consider variational inequalities of the form

$$
\left\{\begin{array}{l}
\langle L(u)+G(u), v-u\rangle+j(v)-j(u) \geq\langle f, v-u\rangle, \quad \forall v \in K, \\
u \in K .
\end{array}\right.
$$

Here $\Omega \subset \mathbb{R}^{N}$ is a bounded domain, $1<p<\infty$ and $\mu$ is the positive measure associated with a $p$-admissible function $m$ in the sense of [20], that is, $d \mu=m(x) d x$, where $d x$ is the usual Lebesgue measure in $\mathbb{R}^{N}$. We denote by $X=H^{1 . p}(\Omega, \mu)$ the (first-order) weighted Sobolev space based on the weighted Lebesgue space $L^{p}(\Omega, \mu)$. Here $X$ is equipped with the usual norm

$$
\|u\|=\|u\|_{1, p}=\|u\|_{L^{p}(\Omega, \mu)}+\|\mid \nabla u\|_{L^{p}(\Omega, \mu)} .
$$

We refer to [20] for more detailed discussions on $p$-admissible weight functions and their related weighted Lebesgue and Sobolev spaces.

We assume in (3.1) that $j$ is a convex, lower semicontinuous functional from $X$ to $\mathbb{R} \cup\{\infty\}$. The typical form of $j$ is either

$$
j(u)=\int_{\Omega} \psi(x, u(x)) d \mu \quad \text { or } \quad j(u)=\int_{\partial \Omega} \psi(x, u(x)) d S,
$$

with $\psi(x, u)$ being a Carathéodory function which is convex in $u$. As usual, $d S$ denotes the surface measure on $\partial \Omega$.

The set $K$ is closed and convex in $H^{1, p}(\Omega, \mu)$ and is typically defined by some obstacles, such as $K=\{u \in X: u \geq \psi$ a.e. on $\Omega\}$, or by certain gradient conditions such as $K=\{u \in X:|\nabla u| \leq c$ a.e. on $\Omega\}$.

The nonlinear operator $L: H^{1, p}(\Omega, \mu) \rightarrow\left[H^{1, p}(\Omega, \mu)\right]^{*}$ is defined by

$$
\langle L(u), v\rangle=\int_{\Omega} A(x, \nabla u) \cdot \nabla v d x,
$$

where $A: \Omega \times \mathbb{B}^{N} \rightarrow \mathbb{R}^{N}$, representing a degenerate elliptic operator, is a Carathéodory function that satisfies the conditions

$$
\left\{\begin{array}{l}
A(x, v) \cdot v \geq \alpha m(x)|v|^{p}, \\
|A(x, v)| \leq \beta m(x)|v|^{p-1}, \quad \text { a.e. } x \in \Omega, \forall v \in \mathbb{R}^{N},
\end{array}\right.
$$

with $\alpha, \beta>0$ (see $[20,31]$ ). 
A typical example of such an operator $L$ is the quasilinear elliptic operator

$$
\langle L(u), v\rangle=\int_{\Omega} \sum_{i=1}^{N}\left(\sum_{j=1}^{N} a_{i j}(x, \nabla u) \partial_{j} u\right) \partial_{i} v d x .
$$

The operator $A$ is therefore given by

$$
A_{i}(x, \xi)=\sum_{j=1}^{N} a_{i j}(x, \xi) \xi_{i} \quad(i=1,2, \ldots, N) .
$$

The degenerate elliptic condition and growth condition in (3.3) are in this case

$$
\sum_{i, j=1}^{N} a_{i j}(x, \xi) \xi_{i} \xi_{j} \geq \alpha m(x)|v|^{p} \text { and } \sum_{i=1}^{N}\left|\sum_{j=1}^{N} a_{i j}(x, \xi) \xi_{i}\right| \leq \beta m(x)|v|^{p-1},
$$

for a.e. $x \in \Omega, \forall v \in \mathbb{R}^{N}$. The latter condition holds if for all $i, j \in\{1, \ldots, N\}$,

$$
\left|a_{i j}(x, \xi)\right| \leq \beta_{0} m(x)|v|^{p-2}, \quad \text { for a.e. } x \in \Omega, \forall v \in \mathbb{R}^{N} .
$$

A particular case of $A$ in (3.4) is the degenerate $p$-Laplacian, that is, $A(x, \xi)=$ $m(x)|\xi|^{p-2} \xi$, for which (3.5) is clearly satisfied. A problem with a degenerate $p$ Laplacian is studied in detail in the next section.

The mapping $G: X \rightarrow X^{*}$ is given by $\langle G(u), v\rangle=\int_{\Omega} g(x, u) v d \mu$, where $g: \Omega \times \mathbb{R} \rightarrow \mathbb{B}$ is a Carathéodory function with the growth condition

$$
|g(x, u)| \leq a(x)+b|u|^{s-1}, \quad(x \in \Omega, u \in \mathbb{R}),
$$

where $1 \leq s \leq p^{*}$ ( $p^{*}$ is the Sobolev critical exponent), $b>0$ and $a \in L^{s^{\prime}}(\Omega, \mu)\left(s^{\prime}\right.$ being the conjugate exponent of $s$ ). Moreover,

$$
\liminf _{|u| \rightarrow \infty} \frac{g(x, u) u}{|u|^{p}} \geq 0, \quad \text { for a.e. } x \in \Omega,
$$

and

$$
g(x, u) u \geq-c_{0}(x)|u|^{p}-d_{0}(x),
$$

for some $d_{0} \in L^{\prime}(\Omega, \mu)$ and $c_{0} \in L^{s /(s-p)}(\Omega, \mu)$.

We note that (3.7) is satisfied if the convergence in (3.6) is uniform with respect to $x \in \Omega$. Another specific case where (3.6) and (3.7) are satisfied is where $g$ can be written as a sum, $g(x, u)=F(x, u)+H(x, u)$, where $F$ and $H$ are Carathéodory functions on $\Omega \times \mathbb{R}$ such that $H$ satisfies the sign condition

$$
H(x, u) u \geq 0,
$$


for a.e. $x \in \Omega$, all $u \in \mathbb{R}$ with $|u|$ large, and $F$ satisfies a sub-( $(p-1)$ growth condition,

$$
F(x, u) u \geq-a_{1}(x)-b_{1}|u|^{\sigma},
$$

where $a_{1} \in L^{1}(\Omega, \mu), a_{1} \geq 0, b_{1} \in \mathbb{R}^{+}$, and $\sigma<p$. In fact, in this case,

$$
\liminf _{|u| \rightarrow \infty} \frac{g(x, u) u}{|u|^{p}} \geq \liminf _{|u| \rightarrow \infty} \frac{F(x, u) u}{|u|^{p}} \geq 0,
$$

by the above growth condition. Moreover, for $|u| \geq C(C>0$ sufficiently large $)$,

$$
g(x, u) u \geq F(x, u) u \geq-a_{1}(x)-b_{1}|u|^{p} .
$$

For $|u| \leq C$, we have

$$
|g(x, u) u| \leq a(x)|u|+b|u|^{s} \leq C a(x)+C^{s} b .
$$

Hence, for all $u \in X$,

$$
g(x, u) u \geq-a_{1}(x)-b_{1}|u|^{p}-C a(x)-C^{s} b,
$$

with $a_{1}+C a+C^{s-1} b \in L^{1}(\Omega, \mu)$. This means that $g$ satisfies (3.6) and (3.7).

We assume hereafter that the measure $d \mu=m d x$ satisfies the condition $m \in$ $L^{\sigma}(\Omega), m^{-1} \in L^{\xi}(\Omega)$, where $\sigma, \xi>1$ and

$$
\begin{cases}1 / \sigma+1 / \xi<p / N, & \text { if } p / N<1+1 / \xi, \\ 1<\sigma \leq \infty, & \text { if } p / N \geq 1+1 / \xi,\end{cases}
$$

which guarantees that the embedding $H^{1, p}(\Omega, \mu) \hookrightarrow L^{p}(\Omega, \mu)$ is compact (see [31, Proposition 1]).

Before stating the main result about the solvability of (3.1), we note that for conditions of Landesman-Lazer type, it has been usually assumed that $G$ has linear growth from below, as in for example $[5,6,11,21,27,34]$. We consider here cases where the perturbing term $g$ has a more general (subcritical) growth condition from below. The results, restricted to second-order, nondegenerate, semi-linear elliptic equations containing the Laplacian, are in the same spirit as the results in [19] and [24]. However, different variational methods are used in those papers, which require variational structures (that is, the existence of potential functionals) of the problems. Our results here therefore give another approach to those problems, which can also be extended to problems without variational structures, to variational inequalities and degenerate problems.

We also observe that the usual theorems of Landesman-Lazer type in [11, 21, 27, 34] and [19] can be obtained in a unified manner using Theorem 1 above.

We now prove the following result about the existence of solutions of (3.1). We identify constant functions with real numbers, and therefore still use $\mathbb{R}$ to denote the set of constant functions. 
THEOREM 2. Assume g satisfies the growth condition (from below)

$$
g(x, u) u \geq-c(x)|u|^{q}-d(x), \quad \text { a.e. } x \in \Omega, \forall u \in \mathbb{R},
$$

where $d \in L^{1}(\Omega, \mu)$ and $c \in L^{s /(s-q)}(\Omega, \mu)(1<q \leq s)$. Let

$$
\left\{\begin{array}{l}
g_{q}^{+}(x)=\liminf _{u \rightarrow \infty} \frac{g(x, u)}{u^{q-1}}, \\
g_{q}^{-}(x)=\limsup _{u \rightarrow-\infty} \frac{g(x, u)}{|u|^{q-1}} .
\end{array}\right.
$$

\section{If either}

(i) rc $K \cap \mathbb{R}=\{0\}$ or

(ii) $1 \in \mathrm{rc} K,-1 \notin \mathrm{rc} K$ (respectively $-1 \in \mathrm{rc} K, 1 \notin \mathrm{rc} K$ ) and $\int_{\Omega} g_{q}^{+} d \mu>0$ (respectively $\int_{\Omega} g_{q}^{-} d \mu<0$ ) or

(iii) $\mathbb{R} \subset$ rc $K$ and

$$
\int_{\Omega} g_{q}^{+} d \mu>0>\int_{\Omega} g_{q}^{-} d \mu
$$

then (3.1) has a solution.

PROOF OF THEOREM 2. We prove the theorem under assumption (iii). The other cases, being simpler, are carried out similarly. We also note that if $K$ is a bounded set, then rc $K=\{0\}$, and (i) is satisfied. Equation (3.1) is of the form (2.2) with $X=H^{1, p}(\Omega, \mu)$ and $A=L+G$. We check the conditions of Theorem 1. First, we verify that $(A, j)$ has Property $(\mathrm{P})$ in $X$, using Proposition 1. By the assumptions on $A$ (see (3.8)), $L$ satisfies (2.12). Assume that $\liminf _{\|u\| \rightarrow \infty}\left(\langle G(u), u\rangle /\|u\|^{p}\right)<0$, that is, there exists a sequence $\left\{u_{n}\right\}$ in $K$ such that $\left\|u_{n}\right\| \rightarrow \infty$ and

$$
\frac{\left\langle G\left(u_{n}\right), u_{n}\right\rangle}{\left\|u_{n}\right\|^{p}} \leq \alpha<0, \quad \forall n .
$$

By passing to a subsequence, we can assume, without loss of generality, that $u_{n} /\left\|u_{n}\right\| \rightarrow w$ in $H^{1 . p}(\Omega, \mu)$. By the compact embedding $H^{1 . p}(\Omega, \mu) \rightarrow L^{p}(\Omega, \mu)$, $u_{n} /\left\|u_{n}\right\| \rightarrow w$ in $L^{p}(\Omega, \mu)$. Passing once more to a subsequence, if necessary, we can find a function $\bar{w} \in L^{p}(\Omega, \mu)$ such that

$$
\left|u_{n}\right| /\left\|u_{n}\right\| \leq \bar{w}, \quad \forall n,
$$

and moreover,

$$
u_{n} /\left\|u_{n}\right\| \rightarrow w \text { a.e. in } \Omega \text {. }
$$


On the other hand,

$$
\frac{\left\langle G\left(u_{n}\right), u_{n}\right\rangle}{\left\|u_{n}\right\|^{p}}=\int_{\Omega} \frac{g\left(x, u_{n}\right) u_{n}}{\left\|u_{n}\right\|^{p}} d \mu=\int_{\Omega} \frac{g\left(x, u_{n}\right) u_{n}}{\left|u_{n}\right|^{p}}\left(\frac{\left|u_{n}\right|}{\left\|u_{n}\right\|}\right)^{p} d \mu .
$$

If $w(x) \neq 0$, then $u_{n}(x)$ is either (strictly) positive or negative for all $n$ sufficiently large (by (3.13)). Since

$$
\left|u_{n}(x)\right|=\left\|u_{n}\right\|\left|\frac{u_{n}(x)}{\left\|u_{n}\right\|}\right| \rightarrow \infty,
$$

we have from (3.6), (3.13) and Fatou's lemma, that

$$
\begin{gathered}
\liminf _{n \rightarrow \infty} \int_{\{x \in \Omega: w(x) \neq 0\}} \frac{g\left(x, u_{n}\right) u_{n}}{\left|u_{n}(x)\right|^{p}}\left(\frac{\left|u_{n}(x)\right|}{\left\|u_{n}\right\|}\right)^{p} d \mu \\
\geq \int_{\{x \in \Omega: w(x) \neq 0\}} \liminf _{n \rightarrow \infty} \frac{g\left(x, u_{n}\right) u_{n}}{\left|u_{n}(x)\right|^{p}} \cdot \lim _{n \rightarrow \infty}\left(\frac{\left|u_{n}(x)\right|}{\left\|u_{n}\right\|}\right)^{p} d \mu \\
\geq \int_{\{x \in \Omega: w(x) \neq 0\}}\left[\liminf _{|u| \rightarrow \infty} \frac{g(x, u) u}{|u|^{p}} \cdot|w(x)|^{p}\right] d \mu \geq 0 .
\end{gathered}
$$

Also, it follows from (3.7) that

$$
\begin{aligned}
\liminf & \int_{\{x \in \Omega: w(x)=0\}} \frac{g\left(x, u_{n}(x)\right) u_{n}(x)}{\left\|u_{n}\right\|^{p}} d \mu \\
& \geq \int_{\{x \in \Omega: w(x)=0\}} \liminf \frac{g\left(x, u_{n}(x)\right) u_{n}(x)}{\left\|u_{n}\right\|^{p}} d \mu \\
& \geq \int_{\{x \in \Omega: w(x)=0\}} \liminf \left(-c_{0}(x) \frac{\left|u_{n}(x)\right|^{p}}{\left\|u_{n}\right\|^{p}}-\frac{d_{0}(x)}{\left\|u_{n}\right\|^{p}}\right) d \mu \\
& =\int_{\{x \in \Omega: w(x)=0\}}-c_{0}(x) \lim \left|\frac{u_{n}(x)}{\left\|u_{n}\right\|}\right|^{p} d \mu=\int_{\{x \in \Omega: w(x)=0\}}|w(x)|^{p} d \mu=0 .
\end{aligned}
$$

We note that Fatou's lemma is applicable in both (3.14) and (3.15), because from (3.7) and (3.12),

$$
\frac{g\left(x, u_{n}\right) u_{n}}{\left\|u_{n}\right\|^{p}} \geq-c_{0} \frac{\left|u_{n}\right|^{p}}{\left\|u_{n}\right\|^{p}}-\frac{d_{0}}{\left\|u_{n}\right\|^{p}} \geq-c_{0} \bar{w}^{p}-d_{0}
$$

and $c_{0} \bar{w}^{p}+d_{0} \in L^{1}(\Omega, \mu)$. Now, in view of (3.14) and (3.15),

$$
\begin{aligned}
\liminf \frac{\left\langle G\left(u_{n}\right), u_{n}\right\rangle}{\left\|u_{n}\right\|^{p}} \geq & \liminf \int_{\mid x: w(x) \neq 0\}} \frac{g\left(x, u_{n}\right) u_{n}}{\left|u_{n}\right|^{p}}\left(\frac{\left|u_{n}\right|}{\left\|u_{n}\right\|}\right)^{p} d \mu \\
& +\liminf \int_{\mid x: w(x)=0\}} \frac{g\left(x, u_{n}\right) u_{n}}{\left\|u_{n}\right\|^{p}} d \mu \\
\geq & 0,
\end{aligned}
$$


contradicting (3.11). This contradiction proves (2.13). On the other hand, for $w \in X$,

Hence

$$
\Phi(w)=\langle L(w), w\rangle=\int_{\Omega} A(x, \nabla w) \cdot \nabla w d x \geq \alpha \int_{\Omega}|\nabla w|^{p} d \mu .
$$

$$
\Phi(w)=0 \Longleftrightarrow|\nabla w|=0 \text { a.e. in } \Omega \Longleftrightarrow w=\text { constant . }
$$

Thus, for all $u \in X, w \in \Phi$,

$$
\Phi(u-w)=\int_{\Omega} A(x, \nabla(u-w)) \cdot \nabla(u-w) d x=\int_{\Omega} A(x, \nabla u) \cdot \nabla u d x=\Phi(u) .
$$

Equation (2.14) results. Now, let $P(u)=\int_{\Omega}|u|^{p} d \mu, u \in X$. It follows from the compact embedding $W^{1 . p}(\Omega, \mu) \hookrightarrow L^{p}(\Omega, \mu)$ that $P$ is completely continuous. Moreover, for all $u \in X$,

$$
\Phi(u)+P(u) \geq \alpha \int_{\Omega}|\nabla w|^{p} d \mu+\int_{\Omega}|u|^{p} d \mu \geq C\|u\|^{p} .
$$

Hence (2.15) is also satisfied.

We have checked all the conditions in Proposition 1 . Thus $(A, j)$ has Property (P). We now verify the compatibility condition in Theorem 1. Assume that (3.10) is satisfied. Let $w \in \operatorname{rc} K$ and $\left\{u_{n}\right\} \subset K$ be such that (2.3)-(2.5) hold. We use (2.4) with $\lambda=q$ and then $\lambda=p$. First, letting $\lambda=p$ in (2.4), we get

$$
\begin{aligned}
0 & \geq \lim \sup \frac{\left\langle A\left(u_{n}\right), u_{n}\right\rangle}{\left\|u_{n}\right\|^{p}} \geq \lim \sup \frac{\left\langle L\left(u_{n}\right), u_{n}\right\rangle}{\left\|u_{n}\right\|^{p}}+\lim \inf \frac{\left\langle G\left(u_{n}\right), u_{n}\right\rangle}{\left\|u_{n}\right\|^{p}} \\
& \geq \limsup \frac{\left\langle L\left(u_{n}\right), u_{n}\right\rangle}{\left\|u_{n}\right\|^{p}} \quad \text { (by (2.13)) } \geq \alpha \limsup \int_{\Omega}\left|\nabla\left(\frac{u_{n}}{\left\|u_{n}\right\|}\right)\right|^{p},
\end{aligned}
$$

by (3.3). Since $w_{n}:=u_{n} /\left\|u_{n}\right\| \rightarrow w$,

$$
0=\lim \left(\int_{\Omega}\left|\nabla w_{n}\right|^{p} d \mu\right)^{1 / p} \geq\left(\int_{\Omega}|\nabla w|^{p} d \mu\right)^{1 / p},
$$

by the weak lower semicontinuity of the semi-norm $\||\nabla u|\|_{L^{p}(\Omega)}$. Thus $|\nabla w|=0$ a.e. in $\Omega$ (note that the sets of measure 0 with respect to $d \mu$ and $d x$ are the same), that is, $w$ is constant. Now, by letting $\lambda=q$ in (2.4), one gets

$$
\begin{aligned}
0 & \geq \limsup \frac{\left\langle A\left(u_{n}\right), u_{n}\right\rangle}{\left\|u_{n}\right\|^{q}}=\lim \sup \left[\frac{\left\langle L\left(u_{n}\right), u_{n}\right\rangle}{\left\|u_{n}\right\|^{q}}+\frac{\left\langle G\left(u_{n}\right), u_{n}\right\rangle}{\left\|u_{n}\right\|^{q}}\right] \\
& \geq \limsup \frac{\left\langle G\left(u_{n}\right), u_{n}\right\rangle}{\left\|u_{n}\right\|^{q}}=\limsup \int_{\Omega} \frac{g\left(x, u_{n}(x)\right) u_{n}(x)}{\left\|u_{n}\right\|^{q}} d \mu .
\end{aligned}
$$


By passing to a subsequence, if necessary, we can assume that $u_{n}(x) /\left\|u_{n}\right\| \rightarrow w(x)$, for a.e. $x \in \Omega$. Since $w \in \mathbb{R}$, we have three cases: $w>0, w<0$ and $w=0$. Assume $w>0$. It follows from (3.15) that for almost all $x \in \Omega, u_{n}(x)>0$ for all $n$ sufficiently large (depending on $x$ ), and moreover, $u_{n}(x) \rightarrow \infty$, for a.e. $x \in \Omega$. Due to the growth condition (3.9), we can use Fatou's lemma to obtain

$$
\begin{aligned}
\liminf _{n \rightarrow \infty} & \int_{\Omega} \frac{g\left(x, u_{n}(x)\right) u_{n}(x)}{\left\|u_{n}\right\|^{q}} d \mu \\
& \geq \int_{\Omega} \liminf _{n \rightarrow \infty}\left\{\frac{g\left(x, u_{n}(x)\right)}{\left[u_{n}(x)\right]^{q-1}}\left[\frac{u_{n}(x)}{\left\|u_{n}\right\|}\right]^{q}\right\} d \mu \\
& \geq \int_{\Omega} \liminf _{n \rightarrow \infty} \frac{g\left(x, u_{n}(x)\right)}{\left[u_{n}(x)\right]^{q-1}} \cdot \lim \left[\frac{u_{n}(x)}{\left\|u_{n}\right\|}\right]^{q} d \mu \\
& \geq \int_{\Omega} \liminf _{u \rightarrow \infty} \frac{g(x, u)}{u^{q-1}} \cdot\left[\lim \frac{u_{n}(x)}{\left\|u_{n}\right\|}\right]^{q} d \mu=w \int_{\Omega} g_{q}^{+}(x) d \mu>0,
\end{aligned}
$$

since $w>0$ and $\int_{\Omega} g_{q}^{+}(x) d \mu \in(0, \infty)$, by (3.10).

It is clear that (3.17) contradicts (3.16). Hence $w$ cannot be positive. Similarly, using the second inequality in (3.10), one can show that $w$ cannot be negative either. Consequently, $w=0$. This implies $w=0 \in \operatorname{rc} K$ and $\left\langle A\left(u_{n}\right), 0\right\rangle=0$. Moreover, $j_{\infty}(0)=0=\langle f, 0\rangle=\min \left\{-j_{\infty}(0), 0\right\}$, that is, $(2.10)$ is satisfied.

We have proved that the compatibility condition in Theorem 1 holds. The existence of a solution of (3.1) now follows from Theorem 1. The theorem is proved in case (iii).

Assume now (i). If $w \in \operatorname{rc} K$ satisfies (2.3)-(2.4), then, as shown previously, $w \in \mathbb{R}$. Hence, $w=0$. The remaining part of the proof is carried out as above. Now, assume the first case of (ii). Since $w \in \mathbb{R} \cap \mathrm{rc} K$, we must have $w \geq 0$. But $w$ cannot be positive as in the proof for case (iii). Thus $w=0$ and we have again the same arguments.

We now consider the classical case of nonlinearity with linear growth from below. We have the following extension of usual results as in $[5,19,24]$ etc. to degenerate equations and inequalities.

THEOREM 3. Assume $g$ satisfies the growth condition $g(x, u) u \geq-c(x)|u|-d(x)$, $x \in \Omega, u \in \mathbb{R}$, where $d \in L^{1}(\Omega, \mu)$ and $c \in L^{s^{\prime}}(\Omega, \mu)$. Let

$$
g_{1}^{+}(x)=\liminf _{u \rightarrow \infty} g(x, u), \quad g_{1}^{-}(x)=\limsup _{u \rightarrow-\infty} g(x, u) .
$$

If either $\mathbb{R} \cap \mathrm{rc} K=\{0\}$, or $1 \in \mathrm{rc} K,-1 \notin \mathrm{rc} K$ and

$$
\int_{\Omega} g_{1}^{+} d \mu+j_{\infty}(1)>\langle f, 1\rangle
$$


or $-1 \in \mathrm{rc} K, 1 \notin \mathrm{rc} K$ and

$$
\int_{\Omega} g_{1}^{-} d \mu-j_{\infty}(-1)<\langle f, 1\rangle
$$

or $\mathbb{R} \subset \operatorname{rc} K$ (that is, $\pm 1 \in \operatorname{rc} K$ ) and

$$
\int_{\Omega} g_{1}^{-} d \mu-j_{\infty}(-1)<\langle f, 1\rangle<\int_{\Omega} g_{1}^{+} d \mu+j_{\infty}(1),
$$

then (3.1) has a solution.

Proof. Again, we apply Theorem 1. As in the proof of Theorem 2, A has Property (P). We only need to check the compatibility condition. This is done in the case where $1 \in \mathrm{rc} K,-1 \notin \mathrm{rc} K$ and (3.18) is satisfied. The proofs for the other cases are similar. Suppose $\left\{u_{n}\right\}$ and $w$ satisfy (2.3)-(2.5) and (2.8). As in the proof of Theorem 2, we can show from these assumptions that $w \in \mathbb{R}$. From (2.8),

Hence

$$
\lim \sup \left[\frac{\left\langle L\left(u_{n}\right), u_{n}\right\rangle}{\left\|u_{n}\right\|}+\frac{\left\langle G\left(u_{n}\right), u_{n}\right\rangle}{\left\|u_{n}\right\|}\right]+j_{\infty}(w) \leq\langle f, w\rangle .
$$

$$
\lim \sup \frac{\left\langle G\left(u_{n}\right), u_{n}\right\rangle}{\left\|u_{n}\right\|}+j_{\infty}(w) \leq\langle f, w\rangle .
$$

Since $w \in \mathbb{R} \cap \mathrm{rc} K$, we must have $w \geq 0$. Assume $w>0$. We have

$$
\liminf \frac{\left\langle G\left(u_{n}\right), u_{n}\right\rangle}{\left\|u_{n}\right\|} \geq \int_{\Omega} \liminf \frac{g\left(x, u_{n}(x)\right) u_{n}(x)}{\left\|u_{n}\right\|} d \mu \geq w \int_{\Omega} g_{1}^{+}(x) d \mu .
$$

It follows from (3.19) and (3.20) that $w \int_{\Omega} g_{1}^{+}(x) d \mu+j_{\infty}(w) \leq\langle f, w\rangle$. From the homogeneity of $j_{\infty}$ and $\langle f, \cdot\rangle$, we have $\int_{\Omega} g_{1}^{+}(x) d \mu+j_{\infty}(1) \leq\langle f, 1\rangle$, contradicting (3.18). Hence $w=0$, for which (2.10) is obviously satisfied. Hence, by Theorem 1, (3.1) has a solution.

3.2. A resonance problem with weighted $p$-Laplacian In this section we present another application of Theorem 1 to a resonance problem for a variational inequality containing a weighted $p$-Laplacian. Consider the variational inequality given by

$$
\left\{\begin{array}{l}
\int_{\Omega}|\nabla u|^{p-2} \nabla u \cdot \nabla(v-u) d \mu-\lambda_{0} \int_{\Omega}|u|^{p-2} u(v-u) d \mu \\
\quad+\int_{\Omega} g(x, u, \nabla u)(v-u) d \mu+j(v)-j(u) \geq 0, \quad \forall v \in K, \\
u \in K .
\end{array}\right.
$$


Here $X=H_{0}^{1}(\Omega, \mu)$ with the usual norm $\|u\|=\|u\|_{H_{0}^{1}(\Omega, \mu)}=\|\mid \nabla u\|_{L^{\rho}(\Omega, \mu)}, K \subset X$ is a closed convex set and $j: X \rightarrow \mathbb{R} \cup\{\infty\}$ is a convex, lower semicontinuous functional, $0 \in K$ and $j(0)=0$.

Also $\lambda_{0}$ is the principal eigenvalue associated with the weighted $p$-Laplacian, defined by

$$
\lambda_{0}=\min _{u \in X \backslash\{0\}} \frac{\int_{\Omega}|\nabla u|^{p} d \mu}{\int_{\Omega}|u|^{p} d \mu}=\min \left\{\int_{\Omega}|\nabla u|^{p} d \mu: \int_{\Omega}|u|^{p} d \mu=1\right\} .
$$

Since the set $S=\left\{u \in X: \int_{\Omega}|u|^{p} d \mu=1\right\}$ is weakly closed and the mapping $u \mapsto \int_{\Omega}|\nabla u|^{p} d \mu=\|u\|_{V}^{p}$ is weakly lower semicontinuous, the minimisation problem in (3.22) has a solution and $\lambda_{0}>0$. By Liusternik's theorem (see for example [26]), $u$ is a solution of (3.22) if and only if $u$ is an eigenvector of the weighted $p$-Laplacian corresponding to $\lambda_{0}$, that is,

$$
\int_{\Omega}|\nabla u|^{p-2} \nabla u \cdot \nabla v d \mu=\lambda_{0} \int_{\Omega}|u|^{p-2} u v d \mu, \quad \forall v \in X .
$$

Assume that $g: \Omega \times \mathbb{B} \times \mathbb{R}^{N} \rightarrow \mathbb{R}$ is a Carathéodory function that satisfies the growth condition $|g(x, u, \xi)| \leq a(x)+b\left(|u|^{s-1}+|\xi|^{s-1}\right), a \in L^{s^{\prime}}(\Omega)$ and $b \geq 0$. Let

$$
\begin{gathered}
\langle L(u), v\rangle=\int_{\Omega}|\nabla u|^{p-2} \nabla u \cdot \nabla v d \mu, \quad\langle B(u), v\rangle=\int_{\Omega}|u|^{p-2} u v d \mu, \\
\langle G(u), v\rangle=\int_{\Omega} g(x, u, \nabla u) v d \mu,
\end{gathered}
$$

and $A=L-\lambda_{0} B+G$. As usual, we denote the set of all eigenvectors corresponding to $\lambda_{0}$ by $E\left(\lambda_{0}\right):=\left(L-\lambda_{0} B\right)$. Equation (3.21) is of the form (2.2) with $f=0$.

We assume that $G$ satisfies condition (2.13). Note that (2.13) is satisfied if $g(x, u, \xi)$ has the growth condition $g(x, u, \xi) u \geq-b_{0}\left(|u|^{p}+|\xi|^{p}\right)-d_{0}(x),\left(b_{0} \geq 0, d_{0} \in\right.$ $\left.L^{1}(\Omega, \mu)\right)$ and $\liminf \sin _{|u| \rightarrow \infty}\left(g(x, u, \xi) u /|u|^{p}\right) \geq 0$, for a.e. $x \in \Omega$.

From the results in [33, Sections 2.5-2.6], we see from the above assumptions that $A$ is an operator of the calculus of variations type. Thus $A$ is pseudo-monotone on $V$. We now check that $(A, j)$ has Property (P). Assume $\left\{u_{n}\right\}$ satisfies (2.3)-(2.6). It follows from (2.4) and (2.13) that

$$
\lim \sup \frac{\left\langle\left(L-\lambda_{0} B\right) u_{n}, u_{n}\right\rangle}{\left\|u_{n}\right\|^{p}} \leq 0 .
$$

Therefore, with $w_{n}=u_{n} /\left\|u_{n}\right\|$,

$$
\begin{aligned}
0 & \geq \limsup \frac{1}{\left\|u_{n}\right\|^{p}}\left[\int_{\Omega}\left|\nabla u_{n}\right|^{p} d \mu-\lambda_{0} \int_{\Omega}\left|u_{n}\right|^{p} d \mu\right] \\
& =\limsup \left[\int_{\Omega}\left|\nabla w_{n}\right|^{p} d \mu-\lambda_{0} \int_{\Omega}\left|w_{n}\right|^{p} d \mu\right] .
\end{aligned}
$$


As $\int_{\Omega}|\nabla u|^{p} d \mu-\lambda_{0} \int_{\Omega}|u|^{p} d \mu \geq 0, \forall u \in X$ (by (3.22)) it follows that

$$
\lim \sup \left[\int_{\Omega}\left|\nabla w_{n}\right|^{p} d \mu-\lambda_{0} \int_{\Omega}\left|w_{n}\right|^{p} d \mu\right]=0 .
$$

From (2.5) and the compact embedding $H^{1, p}(\Omega, \mu) \hookrightarrow L^{p}(\Omega, \mu)$, we have $w_{n} \rightarrow$ $w$ in $L^{p}(\Omega, \mu)$ and thus $\int_{\Omega}\left|w_{n}\right|^{p} d \mu \rightarrow \int_{\Omega}|w|^{p} d \mu$. Hence, by the weak lower semicontinuity of $\|\cdot\|_{H_{0}^{1 . p}(\Omega, \mu)}^{p}$,

$$
\int_{\Omega}|\nabla w|^{p} d \mu \leq \liminf \int_{\Omega}\left|\nabla w_{n}\right|^{p} d \mu=\lambda_{0} \lim \int_{\Omega}\left|w_{n}\right|^{p} d \mu=\lambda_{0} \int_{\Omega}|w|^{p} d \mu .
$$

Again using (3.24), we have $\int_{\Omega}|\nabla w|^{p} d \mu=\lambda_{0} \int_{\Omega}|w|^{p} d \mu$. Hence

$$
\begin{aligned}
\left\|w_{n}\right\|_{H_{0}^{1, p}(\dot{\Omega}, \mu)} & =\left(\int_{\Omega}\left|\nabla w_{n}\right|^{p} d \mu\right)^{1 / p} \rightarrow\left(\lambda_{0} \int_{\Omega}|w|^{p} d \mu\right)^{1 / p} \\
& =\left(\int_{\Omega}|\nabla w|^{p} d \mu\right)^{1 / p}=\|w\|_{H_{0}^{1 . p}(\Omega, \mu)} .
\end{aligned}
$$

Since $\|\cdot\|_{H_{0}^{1 . p}(\Omega, \mu)}$ is uniformly convex (see $\left.[1,20]\right)$, this together with (2.5) implies that

$$
w_{n} \rightarrow w \text { in } H_{0}^{1, p}(\Omega, \mu) .
$$

Now, letting $\lambda=-\left\|u_{n}\right\|$ in (2.6) and dividing both sides of the inequality thus obtained by $\left\|u_{n}\right\|$, we get $1 \leq\left\|w_{n}-w\right\|, \forall n$. This contradicts (3.25) and proves that $(A, j)$ has Property (P).

For $r \geq 0, x \in \Omega, u \in \Omega, \xi \in \mathbb{R}^{N}$, we denote

$$
\underline{g}_{\infty, r}(x, u, \xi)=\inf _{\substack{n \rightarrow \infty \\ u_{n} \rightarrow u \\ \xi_{n} \rightarrow \xi}}\left\{\liminf _{\substack{n \rightarrow \infty \\ n \rightarrow \infty}} \frac{g\left(x, t_{n} u_{n}, t_{n} \xi_{n}\right)}{t_{n}^{r}}\right\}
$$

and

$$
\bar{g}_{\infty, r}(x, u, \xi)=\sup _{\substack{t_{n} \rightarrow \infty \\ a_{n} \rightarrow u \\ \xi_{n} \rightarrow \xi}}\left\{\limsup _{n \rightarrow \infty} \frac{g\left(x, t_{n} u_{n}, t_{n} \xi_{n}\right)}{t_{n}^{r}}\right\} .
$$

Assume now that $g$ satisfies the growth condition (from below)

$$
g(x, u, \xi) u \geq-c(x)|u|^{q}-d(x)|\xi|^{r}-e(x),
$$

where $0 \leq r \leq q \leq p, q \geq 1, e \in L^{1}(\Omega, \mu), c \in L^{s /(s-q)}(\Omega, \mu), d \in L^{s /(s-r)}(\Omega, \mu)$ and $c, d, e \geq 0$. We have the following existence result for solutions of (3.21). 
THEOREM 4. The variational inequality (3.21) has at least one solution, under one of the following conditions:

(a) g satisfies (3.26) with $q=1$ and

$$
\begin{aligned}
& \int_{\{x \in \Omega: w(x)>0\}} \underline{g}_{\infty .0}(x, w, \nabla w) w d \mu \\
& \quad-\int_{\mid x \in \Omega: w(x)<0\}} \bar{g}_{\infty, 0}(x, w, \nabla w) w d \mu+j_{\infty}(w)>0,
\end{aligned}
$$

for all $w \in E\left(\lambda_{0}\right) \backslash\{0\}$.

(b) $g$ satisfies (3.26) with $q>1$ and

$$
\begin{aligned}
\int_{\{x \in \Omega: w(x)>0\}} & \underline{g}_{\infty, q-1}(x, w, \nabla w) w d \mu \\
- & \int_{\{x \in \Omega: w(x)<0)} \bar{g}_{\infty, q-1}(x, w, \nabla w) w d \mu>0,
\end{aligned}
$$

for all $w \in E\left(\lambda_{0}\right) \backslash\{0\}$.

PROOF. We prove (b); the proof of (a) is similar. As observed above, $A$ has Property (P). We check the compatibility condition in Theorem 1. Assume $w \in \operatorname{rc} K$ and $\left\{u_{n}\right\} \subset K$ satisfy (2.3)-(2.5). Letting $\lambda=p$ in (2.4) and using (2.13), we get

$$
\begin{aligned}
0 & \geq \limsup \frac{\left\langle\left(L-\lambda_{0} B+G\right)\left(u_{n}\right), u_{n}\right\rangle}{\left\|u_{n}\right\|^{p}} \\
& \geq \lim \sup \frac{\left\langle\left(L-\lambda_{0} B\right)\left(u_{n}\right), u_{n}\right\rangle}{\left\|u_{n}\right\|^{p}}+\liminf \frac{\left\langle G\left(u_{n}\right), u_{n}\right\rangle}{\left\|u_{n}\right\|^{p}} \\
& \geq \limsup \frac{\left\langle\left(L-\lambda_{0} B\right)\left(u_{n}\right), u_{n}\right\rangle}{\left\|u_{n}\right\|^{p}} .
\end{aligned}
$$

Hence we have (3.23). Using the same arguments as in the proof of Property (P) presented previously, we get

$$
w \in E\left(\lambda_{0}\right)
$$

and

$$
w_{n} \rightarrow w \text { in } H_{0}^{1}(\Omega, \mu) .
$$

Now, letting $\lambda=q$ in (2.4), one gets

$$
0 \geq \lim \sup \frac{\left\langle\left(L-\lambda_{0} B+G\right)\left(u_{n}\right), u_{n}\right\rangle}{\left\|u_{n}\right\|^{q}} \geq \lim \sup \frac{\left\langle G\left(u_{n}\right), u_{n}\right\rangle}{\left\|u_{n}\right\|^{q}} .
$$


On the other hand,

$$
\begin{aligned}
\frac{\left\langle G\left(u_{n}\right), u_{n}\right\rangle}{\left\|u_{n}\right\|^{q}} & =\int_{\Omega} \frac{g\left(x, u_{n}(x), \nabla u_{n}(x)\right) u_{n}(x)}{\left\|u_{n}\right\|^{q}} d \mu \\
& =\int_{\Omega} \frac{g\left(x,\left\|u_{n}\right\| w_{n}(x),\left\|u_{n}\right\| \nabla w_{n}(x)\right) w_{n}(x)}{\left\|u_{n}\right\|^{q-1}} d \mu \\
& =\left(\int_{\{w>0\}}+\int_{\{w<0\}}+\int_{\{w=0\}}\right) \frac{g\left(x,\left\|u_{n}\right\| w_{n}(x),\left\|u_{n}\right\| \nabla w_{n}(x)\right) w_{n}(x)}{\left\|u_{n}\right\|^{q-1}} d \mu
\end{aligned}
$$

Since $w_{n} \rightarrow w$ in $L^{p}(\Omega, \mu)$ and $\nabla w_{n} \rightarrow \nabla w$ in $\left[L^{p}(\Omega, \mu)\right]^{N}$ (see (3.29)), by passing to a subsequence, we can assume without loss of generality that $w_{n}(x) \rightarrow w(x)$, $\nabla w_{n}(x) \rightarrow \nabla w(x)$, a.e. in $\Omega$, and also $\left|w_{n}\right|,\left|\nabla w_{n}\right| \leq h$, for all $n$, with some $h \in L^{p}(\Omega, \mu)$. For $x \in\{w>0\}$, we have $w_{n}(x)>0$ for all $n$ sufficiently large, and therefore

$$
\begin{aligned}
\lim \inf & {\left[\frac{g\left(x,\left\|u_{n}\right\| w_{n}(x),\left\|u_{n}\right\| \nabla w_{n}(x)\right)}{\left\|u_{n}\right\|^{q-1}} w_{n}(x)\right] } \\
& =\liminf \left[\frac{g\left(x,\left\|u_{n}\right\| w_{n}(x),\left\|u_{n}\right\| \nabla w_{n}(x)\right)}{\left\|u_{n}\right\|^{q-1}}\right] \cdot \lim w_{n}(x) \\
& \geq \underline{g}_{\infty . q-1}(x, w(x), \nabla w(x)) w(x) .
\end{aligned}
$$

Similarly, for $x \in\{w<0\}, w_{n}(x)<0$ for $n$ large, and

$$
\begin{aligned}
\lim \inf & {\left[\frac{g\left(x,\left\|u_{n}\right\| w_{n}(x),\left\|u_{n}\right\| \nabla w_{n}(x)\right)}{\left\|u_{n}\right\|^{q-1}} w_{n}(x)\right] } \\
& =\limsup \left[\frac{g\left(x,\left\|u_{n}\right\| w_{n}(x),\left\|u_{n}\right\| \nabla w_{n}(x)\right)}{\left\|u_{n}\right\|^{q-1}}\right] \cdot \lim w_{n}(x) \\
& \geq \bar{g}_{\infty . q-1}(x, w(x), \nabla w(x)) w(x) .
\end{aligned}
$$

If $w(x)=0$, then, from (3.26),

$$
\begin{aligned}
\int_{\{u=0\}} & \frac{g\left(x, u_{n}(x), \nabla u_{n}(x)\right) u_{n}(x)}{\left\|u_{n}\right\|^{q}} d \mu \\
\geq & \int_{\{w=0\}}\left[-c(x) \frac{\left|u_{n}(x)\right|^{q}}{\left\|u_{n}\right\|^{q}}-d(x) \frac{\left|\nabla u_{n}(x)\right|^{r}}{\left\|u_{n}\right\|^{q}}-\frac{e(x)}{\left\|u_{n}\right\|^{q}}\right] d \mu \\
= & -\int_{\{u=0\}} c(x)\left|w_{n}\right|^{q} d \mu-\frac{1}{\left\|u_{n}\right\|^{q-r}} \int_{\{u=0\}} d(x)\left|\nabla w_{n}(x)\right|^{r} d \mu \\
& -\frac{1}{\left\|u_{n}\right\|^{q}} \int_{\left\{u^{\prime}=0\right\}} e(x) d \mu .
\end{aligned}
$$


Since $\nabla w=0$ a.e. on the set $\{w=0\}$, the limits of the terms in the right-hand side of the above inequality are 0 as $n \rightarrow \infty$. Thus

$$
\liminf _{n \rightarrow \infty} \int_{\{w=0\}} \frac{g\left(x, u_{n}(x), \nabla u_{n}(x)\right) u_{n}(x)}{\left\|u_{n}\right\|^{q}} d \mu \geq 0 .
$$

Now (3.30)-(3.32) and Fatou's lemma imply that

$$
\begin{aligned}
\liminf & \frac{\left\langle G\left(u_{n}\right), u_{n}\right\rangle}{\left\|u_{n}\right\|^{q}} \\
\geq & \left(\liminf \int_{\{w>0\}}+\liminf \int_{\{w<0\}}\right) \frac{g\left(x, u_{n}(x), \nabla u_{n}(x)\right) u_{n}(x)}{\left\|u_{n}\right\|^{q}} d \mu \\
\geq & \int_{\{w>0\}} \liminf \left[\frac{g\left(x, u_{n}(x), \nabla u_{n}(x)\right) u_{n}(x)}{\left\|u_{n}\right\|^{q}}\right] d \mu \\
& +\int_{\{w<0\}} \liminf \left[\frac{g\left(x, u_{n}(x), \nabla u_{n}(x)\right) u_{n}(x)}{\left\|u_{n}\right\|^{q}}\right] d \mu \\
\geq & \int_{\{w>0\}} \underline{g}_{\infty, q-1}(x, w, \nabla w) w d \mu+\int_{\{w<0\}} \bar{g}_{\infty, q-1}(x, w, \nabla w) w d \mu .
\end{aligned}
$$

Hence

$$
0 \geq \int_{\{w>0\}} \underline{g}_{\infty, q-1}(x, w, \nabla w) w d \mu+\int_{\{w<0\}} \bar{g}_{\infty, q-1}(x, w, \nabla w) w d \mu .
$$

Together with (3.27) and (3.28), this implies that $w=0$, for which (2.9) and (2.10) are obviously satisfied. By Theorem 1, (3.21) has a solution. The proof of (a), in which we use (2.8) instead of (2.4), is similar and is thus omitted.

An immediate consequence of Theorem 4 , in the case $p=q$, is the following.

COROLLARY 1. Assume $q=p$ in (3.26) and that

$$
E\left(\lambda_{0}\right)=\left\{\lambda \phi_{0}: \lambda \in \mathbb{R}\right\},
$$

where

$$
\phi_{0}(x)>0, \text { for a.e. } x \in \Omega \text {. }
$$

Then a sufficient condition for (3.27) to be solvable is

and

$$
\underline{g}_{\infty, p-1}\left(x, \phi_{0}, \nabla \phi_{0}\right) \geq 0, \quad \underline{g}_{\infty, p-1}\left(x, \phi_{0}, \nabla \phi_{0}\right) \not \equiv 0 \quad \text { in } \Omega,
$$

$$
\bar{g}_{\infty, p-1}\left(x, \phi_{0}, \nabla \phi_{0}\right) \leq 0, \quad \bar{g}_{\infty, p-1}\left(x, \phi_{0}, \nabla \phi_{0}\right) \not \equiv 0 \quad \text { in } \Omega
$$


REMARK 2. (a) Equations (3.33)-(3.34) are a well-known property of $\lambda_{0}$ when $d \mu=d x$ is the Lebesgue measure (see [4] or [37]).

(b) Corollary 1 is a nonvariational analogue and extension of certain results in [24] and [19]. In those papers, the authors consider the case where $p=2, d \mu=d x$ and $g$ does not depend on $\nabla u$. Hence the problem has a variational structure (that is, the solutions of the equation are critical points of some potential functional). Their condition is on the potential functional $G$ of $g$, rather than on the function $g(x, u) u$. Hence our results stated above (in the particular case where $p=2$ and $d \mu$ is the Lebesgue measure) are the nonvariational counterpart of the cited theorems in [24] and [19]. The results presented here are also valid in other general situations, where the equations or inequalities do not have variational structures. As considered above, an example of such a situation is when the perturbing term also depends on the gradient of the unknown function.

The investigation of minimisation problems by a different approach, concerning equations and variational inequalities with variational structures, will be the subject of a forthcoming paper.

(c) Theorem 4 also shows that the cases $q<p$ and $q=p$ can be proved at the same time, hence unifying Theorem 3 and [19, Corollary 2]. The condition in Theorem 4 (a) is usually referred to as a Landesman-Lazer type condition. Theorem 4 shows that the Landesman-Lazer condition and the conditions in [19] and [24] have the same nature and can thus be treated in the same way, using the recession approach in Theorems 1 and 4. In Theorem 4, when defining the limits, we do not require uniform convergence as in $[19,24]$, but only pointwise convergence with certain growth conditions.

\section{Acknowledgements}

The author would like to thank the referees for the supplementary references and for their valuable comments and suggestions. This work was supported in part by a grant from the University of Missouri Research Board.

\section{References}

[1] R. Adams, Sobolev spaces (Academic Press, New York, 1975).

[2] A. Alvino and G. Trombetti, "Sulle migliori di maggiorazione per une classe di equazioni ellittiche degeneri", Ric. Mat. 27 (1978) 413-428.

[3] A. Alvino and G. Trombetti, "Su une classe di equazioni ellittiche non lineari degeneri", Ric. Mat. 29 (1980) 193-212.

[4] A. Anane, "Simplicité et isolation de la première valeur propre du $p$-Laplacien", C. R. Acad. Sci Paris 305 (1987) 725-728. 
[5] D. D. Ang, K. Schmitt and V. K. Le, "Noncoercive variational inequalities: Some applications", Nonlinear Analysis, TMA 15 (1990) 497-512.

[6] D. D. Ang, K. Schmitt and V. K. Le, "P-coercive variational inequalities and unilateral problems for von Karman's equations", WSSIAA 1 (1992) 15-29.

[7] G. Aronsson, L. C. Evans and Y. Wu, "Fast/slow diffusion and growing sandpiles", J. Differential Equations 131 (1996) 304-335.

[8] C. Baiocchi, G. Buttazzo, F. Gastaldi and F. Tomarelli, "General existence theorems for unilateral problems in continuum mechanics", Arch. Rational Mech. Anal. 100 (1988) 149-189.

[9] C. Baiocchi and A. Capelo, Variational and quasivariational inequalities: applications to free boundary problems (Wiley, New York, 1984).

[10] H. Brézis, "Equations et inéquations non linéaires dans les espaces vectoriels en dualité", Ann. Inst. Fourier 18 (1968) 115-175.

[11] H. Brézis, "Problèmes unilatéraux", J. Math. Pures Appl. 51 (1972) 1-168.

[12] G. R. Cirmi and M. M. Porzio, " $L$-solutions for some nonlinear degenerate elliptic and parabolic equations", Ann. Mat. Pura Appl. 169 (1995) 67-86.

[13] J. I. Diaz, Nonlinear partial differential equations and free boundaries. Vol. 1: Elliptic equations, Research Notes in Math. 106 (Longman, London, 1985).

[14] P. Drabek, A. Kufner and F. Nicolosi, "On the solvability of degenerated quasilinear elliptic equations of higher order", J. Diff. Equations 109 (1994) 325-347.

[15] P. Drabek, A. Kufner and F. Nicolosi, Quasilinear elliptic equations with degenerations and singularities (Walter de Gruyter, Berlin, 1997).

[16] P. Drabek and F. Nicolosi, "Existence of bounded solutions for some degenerated quasilinear elliptic equations", Ann. Mat. Pura Appl. 165 (1993) 217-238.

[17] P. Drabek and F. Nicolosi, "Solvability of degenerate elliptic problems of higher order via LerayLions theorem", Hiroshima Math. J. 26 (1996) 79-90.

[18] G. Duvaut and J. L. Lions, Les inéquations en mécanique et en physique (Dunod, Paris, 1972).

[19] A. Fonda and J.-P. Gossez, "Semicoercive variational problems at resonance: an abstract approach", Differential Integral Equations 3 (1990) 695-708.

[20] J. Heinonen, T. Kilpeläinen and O. Martio, Nonlinear potential theory for degenerate elliptic equations (Cambridge Univ. Press, Cambridge, 1993).

[21] P. Hess, "On the solvability of nonlinear elliptic boundary value problems", Indiana Univ. Math. J. 25 (1976) 461-466.

[22] Y. Huang, "Existence of positive solutions for a class of the $p$-Laplace equations", J. Austral. Math. Soc. Ser. B 36 (1994) 249-264.

[23] A. V. Ivanov and P. Z. Mkrtycjan, "On the solvability of the first boundary value problem for certain classes of degenerating quasilinear elliptic equations of the second order", Proc. Steklov Inst. Math. 147 (1981) 11-35.

[24] J. R. Ward J. Mawhin and M. Willem, "Variational methods and semilinear elliptic equations", Arch. Rational Mech. Anal. 95 (1986) 269-277.

[25] D. Kinderlehrer and G. Stampacchia, An introduction to variational inequalities and their applications (Academic Press, New York, 1980).

[26] M. A. Krasnosels'kii, Topological methods in the theory of nonlinear integral equations (Pergamon Press, Oxford, 1963).

[27] E. M. Landesman and A. C. Lazer, "Nonlinear perturbations of linear elliptic boundary value problems at resonance", J. Math. Mech. 19 (1969/1970) 609-623.

[28] V. K. Le, "Some existence results for noncoercive nonconvex minimization problems with fast or slow perturbing terms", Num. Func. Anal. Optim. 20 (1999) 37-58.

[29] V. K. Le and K. Schmitt, "Minimization problems for noncoercive functionals subject to constraints”, Trans. Amer. Math. Soc. 347 (1995) 4485-4513. 
[30] V. K. Le and K. Schmitt, "Minimization problems for noncoercive functionals subject to constraints, Part II", Adv. Differential Equations 1 (1996) 453-498.

[31] V. K. Le and K. Schmitt, "On boundary value problems for degenerate quasilinear elliptic equations and inequalities", J. Differential Equations 144 (1998) 170-218.

[32] S. Leonardi, "Solvability of degenerate quasilinear elliptic equations", Nonlinear Analysis, TMA 26 (1996) 1053-1060.

[33] J. L. Lions, Quelques méthodes de résolution des problèmes aux limites non linéaires (Dunod, Paris, 1969).

[34] P. J. McKenna and J. Rauch, "Strongly nonlinear perturbations of nonnegative boundary value problems with kernel", J. Differential Equations 28 (1978) 253-265.

[35] M. K. V. Murthy and G. Stampacchia, "Boundary value problems for some degenerate elliptic operators", Ann. Math. Pura Appl. 80 (1968) 1-122.

[36] B. Opic and A. Kufner, Hardy-type inequalities, Pitman Research Notes in Math. 219 (Longman, Harlow, 1990).

[37] M. Ôtani and T. Teshima, "On the first eigenvalue of some quasilinear elliptic equations", Proc. Japan Acad. 64 (1988) 8-10.

[38] L. Prigozhin, "Quasivariational inequality describing the shape of a poured pile", Vychisl. Mat. Mat. Fiz. 26 (1986) 1072-1080.

[39] L. Prigozhin, "Sandpiles and river networks: extended systems with nonlocal interactions", Phys. Rev. E 49 (1994) 1161-1167,

[40] L. Prigozhin, "Variational model of sandpile growth", European J. Appl. Math. 7 (1996) 225-235.

[41] R. T. Rockafellar, "Level sets and continuity of conjugate convex functions", Trans. Amer. Math. Soc. 123 (1966) 46-63.

[42] T. R. Rockafellar, Convex analysis (Princeton University Press, Princeton, NJ, 1970).

[43] N. Trudinger, "Linear elliptic operators with measurable coefficients", Ann. Scuola Norm. Sup. Pisa 27 (1973) 265-308. 